PROCEEDINGS OF THE

AMERICAN MATHEMATICAL SOCIETY

Volume 132, Number 8, Pages 2205-2213

S 0002-9939(04)07386-

Article electronically published on March 10, 2004

\title{
NOTE ON THE STABILITY OF PRINCIPAL BUNDLES
}

\author{
DONGHOON HYEON AND DAVID MURPHY
}

(Communicated by Michael Stillman)

\begin{abstract}
We compare various notions of stability for principal bundles, and show that over a compact Riemann surface of genus greater than 2 , there exist principal SL(2)-bundles that are Ad-stable.
\end{abstract}

\section{INTRODUCTION}

The moduli space of stable principal $G$-bundles over a compact Riemann surface was constructed by A. Ramanathan in his thesis [6]. The method was generalized recently by the first named author in [5], where he gave a construction of a quasiprojective moduli scheme for principal bundles over a projective scheme of arbitrary dimension. However, the moduli constructed in 55 may fail to include some bundles that we would like it to have. For instance, over a compact Riemann surface, the stability condition used in [5] is strictly stronger than the stability employed by Ramanathan. It has thus been of concern to the authors whether a principal bundle that is stable in the sense of [5] exists at all. The purpose of this article is to give an answer to this question, and to give a detailed account of the relation between the various stability conditions.

In section 2, we introduce the stability conditions for principal bundles used by A. Ramanathan, D. Hyeon (Ad-stability), and D. Mumford (for $G=\mathrm{GL}(n)$ ). We give an elementary proof that for $G=\mathrm{GL}(n)$, the stability condition defined by Ramanathan is equivalent to the slope stability of Mumford. We also show that the Ad-stability is strictly stronger than Ramanathan's stability, and that there are no Ad-stable principal $G$-bundles if $G$ is not semisimple. (This is essentially proved in 6].)

In section 3, we briefly recall the main result in 4], and using it, construct examples of Ad-stable SL(2)-bundles. Furthermore, we give an example of an SL(2)bundle that is stable in the sense of Ramanathan, but is not Ad-stable.

Unless otherwise stated, $X$ will be a compact Riemann surface of genus $g$. The letter $G$ will be reserved to denote a connected reductive complex holomorphic Lie group, whose Lie algebra will be denoted $\mathfrak{g}$. For any vector bundle $E$ over $X$, the degree $\operatorname{deg}(E)$ of $E$ is defined by the Riemann-Roch formula

$$
\chi(E)=\operatorname{deg}(E)+\operatorname{rk}(E) \cdot(1-g),
$$

and the slope $\mu(E)$ is the quantity $\frac{\operatorname{deg}(E)}{\operatorname{rk}(E)}$.

Received by the editors February 18, 2002 and, in revised form, February 18, 2003.

2000 Mathematics Subject Classification. Primary 14D20. 


\section{StABILITY NOTIONS FOR PRINCIPAL BUNDLES}

Definition 1. (1) Let $E$ be a principal $G$-bundle over $X$, and $\rho: G \rightarrow H$ a homomorphism of holomorphic complex Lie groups. Let $G$ act diagonally on $E \times H$ by the natural right action on $E$, and through $\rho$ on $H$. It is well known that the quotient bundle $(E \times H) / G$ exists as a principal $H$-bundle, and we denote it by $E_{\rho}(H)$, or by $E(H)$ when the representation used is clear. In a similar fashion, if $F$ is any $G$-space, let $E(F)$ denote the fibre bundle $(E \times F) / G$ with fibre $F$, where $G$ acts diagonally on $E \times F$.

(2) A reduction $\sigma$ of the structure group of a principal $G$-bundle $E$ to a subgroup $P$ of $G$ is a section $\sigma: X \rightarrow E / P$ of the fibre bundle $E / P=E(G / P)$. Then $\sigma^{*} E$ is a $P$-bundle over $X$ and there is a natural isomorphism of $G$-bundles $\sigma^{*} E(G) \simeq E$.

Ramanathan's definition of stability for principal bundles can be phrased in many different ways (e.g., Theorem 2.2. of [2], and [1]). The original version reads as follows.

Definition 2. A principal $G$-bundle $E$ over $X$ is said to be semistable if for every reduction $\sigma: X \rightarrow E / P$ of the structure group to a maximal parabolic $P \subset G$, the following inequality holds:

$$
\operatorname{deg} \sigma^{*} T_{E / P} \geq 0 \text {. }
$$

We call $E$ stable if the above inequality is always strict.

Here, $T_{E / P}$, the tangent bundle over $E / P$ along the fibre of $E$, is defined to be the vector bundle $E(\mathfrak{g} / \mathfrak{p})=(E \times(\mathfrak{g} / \mathfrak{p})) / P$ over $E / P$ associated to the $P$-bundle $E \rightarrow E / P$ and the $P$ action on $\mathfrak{g} / \mathfrak{p}$ ( $\mathfrak{p}$ is the Lie algebra of $P$ ) induced by the adjoint representation.

This stability condition is consistent with the condition defined by Mumford, in the case $G=\mathrm{GL}(n)$. This is not hard to see, but we include an elementary proof for the sake of completeness.

Let $P \subset \mathrm{GL}(n)$ be a parabolic subgroup consisting of matrices of the form

$$
\left(\begin{array}{ll}
A & B \\
0 & C
\end{array}\right)
$$

where $A \in \mathrm{GL}(r)$ and $C \in \mathrm{GL}(n-r)$, with respect to a fixed basis of $\mathbb{C}^{n}$. Let $\sigma: X \rightarrow E / P$ be a reduction of the structure group, and let

$$
\left(\begin{array}{cc}
h_{\alpha \beta} & b_{\alpha \beta} \\
0 & q_{\alpha \beta}
\end{array}\right)
$$

be the transition functions for the $P$-bundle $\sigma^{*} E$ with respect to a trivializing cover $\left\{U_{\alpha}\right\}$. Let $F$ be the rank $r$ subbundle of $E\left(\mathbb{C}^{n}\right)$ corresponding to $\left(h_{\alpha \beta}\right)$. Then the quotient bundle $E\left(\mathbb{C}^{n}\right) / F$ corresponds to $\left(q_{\alpha \beta}\right)$, and we have the following natural isomorphism.

\section{Proposition 1.}

$$
\sigma^{*} T_{E / P} \simeq F^{*} \otimes\left(E\left(\mathbb{C}^{n}\right) / F\right) .
$$

Proof. The adjoint action of $P$ on the Lie algebra $\mathfrak{g l}(n)$ is given by the matrix multiplication

$$
\left(\begin{array}{ll}
h & b \\
0 & q
\end{array}\right) \cdot\left(\begin{array}{ll}
A & B \\
C & D
\end{array}\right) \cdot\left(\begin{array}{cc}
h^{-1} & b^{\prime} \\
0 & q^{-1}
\end{array}\right)=\left(\begin{array}{cc}
* & * \\
q \cdot C \cdot h^{-1} & *
\end{array}\right) .
$$


Here, $\left(\begin{array}{cc}h^{-1} & b^{\prime} \\ 0 & q^{-1}\end{array}\right)$ is the inverse matrix of $\left(\begin{array}{cc}h & b \\ 0 & q\end{array}\right)$. The places with $*$ are covered by $\mathfrak{p}$, and the lower left corner is the only part relevant to $\mathfrak{g} / \mathfrak{p}$. Hence we conclude that the adjoint action of $P$ on $\mathfrak{g} / \mathfrak{p}$ is given by

$$
\left(\left(\begin{array}{cc}
h & b \\
0 & q
\end{array}\right), C\right) \mapsto q \cdot C \cdot h^{-1}
$$

Therefore, the transition functions for $\sigma^{*} E(\mathfrak{g} / \mathfrak{p})$ are $\left({ }^{T} h_{\alpha \beta}^{-1} \otimes q_{\alpha \beta}\right)$, which correspond to $F^{*} \otimes\left(E\left(\mathbb{C}^{n}\right) / F\right)$. (See, for instance, section 0.5 of $[3]$.)

Corollary 1. A principal $\mathrm{GL}(n)$-bundle $E$ over $X$ is semistable (resp. stable) if and only if for every subbundle $F$ of $E\left(\mathbb{C}^{n}\right), \mu(F) \leq \mu\left(E\left(\mathbb{C}^{n}\right)\right)\left(\right.$ resp. $\left.\mu(F)<\mu\left(E\left(\mathbb{C}^{n}\right)\right)\right)$ holds, i.e., $E\left(\mathbb{C}^{n}\right)$ is semistable (resp. stable) in the sense of Mumford.

Proof. We shall prove the statement for the "semistable" part only. One proves the "stable" part by simply replacing every inequality by a strict inequality.

Suppose that $E\left(\mathbb{C}^{n}\right)$ is semistable in the sense of Mumford, and let $\sigma: X \rightarrow E / P$ be a reduction of the structure group to a maximal parabolic subgroup $P$ of $G$. Then $P$ corresponds to a two-step flag

$$
0=V_{0} \subset V_{1} \subset V_{2}=\mathbb{C}^{n} .
$$

Let $F=\sigma^{*}\left(E\left(V_{1}\right)\right)$. By Proposition 1, $\sigma^{*} T_{E / P} \simeq F^{*} \otimes\left(E\left(\mathbb{C}^{n}\right) / F\right)$. Since $E\left(\mathbb{C}^{n}\right)$ is Mumford semistable, we have $\mu(F) \leq \mu\left(E\left(\mathbb{C}^{n}\right)\right)$, which is equivalent to $\mu(F) \leq$ $\mu\left(E\left(\mathbb{C}^{n}\right) / F\right)$. This is in turn equivalent to $\operatorname{deg} F^{*} \otimes\left(E\left(\mathbb{C}^{n}\right) / F\right) \geq 0$, since

$$
\begin{aligned}
\operatorname{deg} F^{*} \otimes\left(E\left(\mathbb{C}^{n}\right) / F\right) & =-\operatorname{deg} F \cdot \operatorname{rk}\left(E\left(\mathbb{C}^{n}\right) / F\right)+\operatorname{rk} F \cdot \operatorname{deg} E\left(\mathbb{C}^{n}\right) / F \\
& =\left(\mu\left(E\left(\mathbb{C}^{n}\right) / F\right)-\mu(F)\right) \cdot \operatorname{rk}\left(E\left(\mathbb{C}^{n}\right) / F\right) \cdot \operatorname{rk}(F) .
\end{aligned}
$$

Therefore, $E$ is semistable as a principal $\operatorname{GL}(n)$-bundle.

Conversely, let $E$ be a semistable principal GL $(n)$-bundle. Any vector subbundle $F$ of $E\left(\mathbb{C}^{n}\right)$ is of the form $\sigma^{*}(E(V))$ for some reduction $\sigma$ of the structure group to a parabolic subgroup $P$ corresponding to a flag $0=V_{0} \subset V \subset \mathbb{C}^{n}$. Since $E$ is stable as a principal $\mathrm{GL}(n)$-bundle, we have

$$
\operatorname{deg} \sigma^{*}\left(T_{E / P}\right)=\operatorname{deg}\left(F^{*} \otimes\left(E\left(\mathbb{C}^{n}\right) / F\right)\right) \geq 0 .
$$

By $(*)$, we know that this implies $\mu(F) \leq \mu\left(E\left(\mathbb{C}^{n}\right)\right)$.

Now we introduce a very important lemma that rephrases the stability condition for principal bundles in terms of the well-known stability condition for vector bundles. This suggests that one may deduce the existence of a moduli space of principal bundles from the existence of a moduli space of vector bundles, which is exactly what Ramanathan did in his thesis [6].

Lemma 1 ([6]). A holomorphic principal G-bundle $E$ over $X$ is semistable if and only if the associated vector bundle $\operatorname{Ad}(E)=(E \times \mathfrak{g}) / G$ is a semistable vector bundle.

In fact, this is taken as the definition of semistability in [2]. Based on this lemma, we make the following definition.

Definition 3. A holomorphic principal $G$-bundle over $X$ is said to be Ad-stable if the associated vector bundle $\operatorname{Ad}(E)$ is a stable vector bundle. 
In [5], the first named author constructed a quasi-projective moduli scheme for Ad-stable principal $G$-bundles over a projective scheme of arbitrary dimension. (The term "Ad-stable" is not used in [5].) It is natural to ask what the relationship is between Ad-stability and stability according to Ramanathan.

Proposition 2. If a principal G-bundle $E$ over a compact Riemann surface $X$ is Ad-stable, then it is stable in the sense of Ramanathan.

Proof. Suppose that $E$ is an Ad-stable $G$-bundle. Since $G$ is reductive, there exists a nondegenerate $G$-invariant bilinear form on $\mathfrak{g}$. Via this form, $\operatorname{Ad}(E)=E(\mathfrak{g})$ is dual to itself and hence is of degree zero. Let $P$ be a parabolic subgroup of $G$ and let $\mathfrak{p}$ be its Lie algebra. Also, let $\sigma: X \rightarrow E / P$ be a reduction of the structure group to $P$. We have the exact sequence of $P$-modules (the module structures are given by the adjoint representation)

$$
0 \rightarrow \mathfrak{p} \rightarrow \mathfrak{g} \rightarrow \mathfrak{g} / \mathfrak{p} \rightarrow 0
$$

and the exact sequence of vector bundles

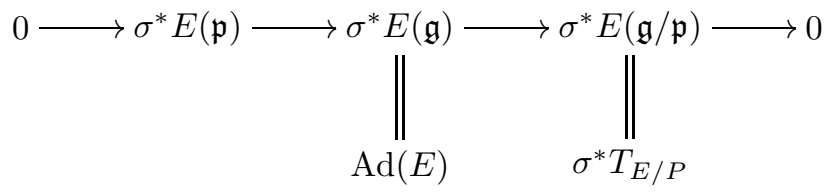

Since $\operatorname{Ad}(E)$ is a stable vector bundle of degree zero, it follows that $\operatorname{deg} \sigma^{*} T_{E / P}>0$, and we are done.

However, as remarked in 2], $\operatorname{Ad}(E)$ can be strictly semistable even if $E$ is stable in the sense of Ramanathan. For example, for any $n>1$, no $\operatorname{GL}(n)$-bundle is Ad-stable, since $\operatorname{Ad}(E)=\operatorname{End}\left(E\left(\mathbb{C}^{n}\right)\right)$ has the trivial line subbundle generated by the trivial section $I d$ defined by $I d_{x}(v)=v$ for all $x \in X$ and $v \in E\left(\mathbb{C}^{n}\right)_{x}$. In fact, we know that

$$
\operatorname{End}\left(E\left(\mathbb{C}^{n}\right)\right)=\operatorname{End}_{o}\left(E\left(\mathbb{C}^{n}\right)\right) \oplus \mathcal{O}_{X},
$$

where $\operatorname{End}_{o}\left(E\left(\mathbb{C}^{n}\right)\right)$ is the bundle of trace-free endomorphisms. This decomposition follows from the fact that there exists a split exact sequence of GL $(n)$-modules

$$
0 \longrightarrow \mathfrak{s l}(n) \longrightarrow \mathfrak{g l}(n) \underset{\iota}{\stackrel{T r}{\rightleftarrows}} \mathbb{C} \longrightarrow 0
$$

where the module structures are given by the adjoint representation. Here $\operatorname{Tr}$ is the trace map and $\iota$ is defined by $\iota(z)=\frac{z}{n} 1,1$ being the identity matrix in $\mathfrak{g l}(n)$. More generally,

Lemma 2. There are no Ad-stable principal $G$-bundles if $G$ is not semisimple.

Proof. Let $\mathfrak{z}$ denote the center of $\mathfrak{g}$. Since we are in characteristic 0 , note that $G$ being semisimple, the connected center $Z^{\circ}(G)$ being trivial, and $\mathfrak{z}=0$ are all equivalent. Suppose that there exists an Ad-stable $G$-bundle $E$. By Proposition 2 $E$ is stable. So we can use Proposition 3.2 of $[\underline{6}]$ to conclude that $H^{0}(X, E(\mathfrak{g}))=\mathfrak{z}$. If $\mathfrak{z} \neq 0$, i.e., if $G$ is not semisimple, then $E(\mathfrak{g})$ has a global section $\phi: \mathcal{O}_{X} \rightarrow E(\mathfrak{g})$ that generates $\mathcal{O}_{X}$ as a subsheaf of $E(\mathfrak{g})$. This contradicts the assumption that $E$ is Ad-stable. 
This shows that the construction in [5] is effective only for semisimple groups. However, even in the semisimple case, stability and Ad-stability are not equivalent. In section 4, we shall encounter an example of a stable $\mathrm{SL}(2)$-bundle that is not Ad-stable.

\section{Ad-Stable SL(2)-Bundles over a Riemann surface}

First we recall the main result in [4]. The fundamental group of $X$ is generated by $2 g$ elements $a_{1}, b_{1}, \ldots, a_{g}, b_{g}$ with the single relation

$$
\prod_{i=1}^{g}\left[a_{i}, b_{i}\right]=a_{1} b_{1} a_{1}^{-1} b_{1}^{-1} \cdots a_{g} b_{g} a_{g}^{-1} b_{g}^{-1}=1 .
$$

We denote this group by $\Gamma_{g}$.

Let $Y$ denote the universal cover of $X$. It is a principal $\Gamma_{g}$-bundle over $X$ (or, a Galois covering of $X$ with Galois group $\Gamma_{g}$ ). Therefore, any rational representation $\rho: \Gamma_{g} \rightarrow \operatorname{GL}(n)$ produces a rank $n$ vector bundle $\left(Y \times \mathbb{C}^{n}\right) / \Gamma_{g}$ over $X$. The main result of $[4$ is the following theorem.

Theorem 1. Let $X$ be a compact Riemann surface of genus $\geq 2$. Then a holomorphic vector bundle of degree zero on $X$ is stable if and only if it arises from an irreducible unitary representation of the fundamental group of $X$.

We need the following basic lemma, which can easily be seen when one considers the transition functions of the bundles involved.

Lemma 3. Let $E$ be a principal $G_{1}$-bundle, and let $\rho_{1}: G_{1} \rightarrow G_{2}$ and $\rho_{2}: G_{2} \rightarrow G_{3}$ be homomorphisms of holomorphic complex Lie groups. Then we have a natural isomorphism

$$
\left(E_{\rho_{1}}\left(G_{2}\right)\right)_{\rho_{2}}\left(G_{3}\right) \simeq E_{\rho_{2} \circ \rho_{1}}\left(G_{3}\right) .
$$

A principal SL(n)-bundle can be thought of as a rank $n$ vector bundle with trivial determinant. Therefore we can immediately deduce the following proposition from Theorem 1 and Lemma 3 .

Proposition 3. Let $X$ be a compact Riemann surface of genus $\geq 2$. Then a holomorphic principal $\mathrm{SL}(n)$-bundle $E$ is Ad-stable if and only if it arises from a representation $\rho: \Gamma_{g} \rightarrow \mathrm{SL}(n)$ such that $\operatorname{ad}(\rho)$, the composition of the adjoint representation ad $: \operatorname{SL}(n) \rightarrow \operatorname{GL}(\mathfrak{s l}(n))$ and $\rho$, is irreducible and unitary.

Remark 1 . Such a $\rho$ is necessarily irreducible and unitary by Lemma 6, which will be shown later in this article. Therefore we conclude that a principal SL $(n)$-bundle is stable if it is Ad-stable. Of course, this also follows from Proposition 2.

We let Ad (resp. ad) denote the adjoint representation $\mathrm{GL}(n) \rightarrow \mathrm{GL}(\mathfrak{g l}(n))$ (resp. $\mathrm{SL}(n) \rightarrow \mathrm{GL}(\mathfrak{s l}(n)))$. Hence for any $x \in \mathrm{SL}(n), \operatorname{Ad}(x)$ denotes the adjoint action of $x$ on $\mathfrak{g l}(n)$, and $\operatorname{ad}(x)$ denotes the adjoint action on $\mathfrak{s l}(n) \subset \mathfrak{g l}(n)$. Let $\left\{e_{1}, \ldots, e_{n}\right\}$ denote the standard basis of $\mathbb{C}^{n}$. Then the standard basis for $\mathfrak{g l}(n)$ consists of the matrices $e_{i j}=e_{i} \otimes e_{j}^{*}$, the matrix with 1 at the $i j$-th place and 0 elsewhere.

Definition 4. Let $G$ be a group and $\rho: G \rightarrow \operatorname{GL}(n)$ a representation. Then $\rho$ is said to be unitary if $\rho(G) \subset \mathrm{U}(n)$. 
Lemma 4. Let $x \in U(n)$. With respect to the basis $\left\{e_{i} \otimes e_{j}^{*}\right\}$, we have

$$
(\operatorname{Ad}(x))_{k l i j}=x_{k i} \bar{x}_{l j} \text {. }
$$

That is,

$$
\operatorname{Ad}(x)\left(e_{i j}\right)=\sum_{k, l} x_{k i} \bar{x}_{l j} e_{k l} .
$$

Proof. Let $x^{\prime}=\bar{x}^{T}$. The adjoint action is given by

$$
\begin{aligned}
& \operatorname{Ad}(x)\left(e_{i j}\right)=x \cdot e_{i} \otimes e_{j}^{*} \cdot x^{\prime} \\
& =\left(\begin{array}{ccccccc}
0 & 0 & \cdots & 0 & x_{1 i} & 0 & \cdots 0 \\
0 & 0 & \cdots & 0 & x_{2 i} & 0 & \cdots 0 \\
\vdots & \vdots & \vdots & \vdots & \vdots & \vdots & \vdots \\
0 & 0 & \cdots & 0 & x_{n i} & 0 & \cdots 0
\end{array}\right) \cdot x^{\prime} \\
& =\left(\begin{array}{cccc}
x_{1 i} x_{j 1}^{\prime} & x_{1 i} x_{j 2}^{\prime} & \cdots & x_{1 i} x_{j n}^{\prime} \\
x_{2 i} x_{j 1}^{\prime} & x_{2 i} x_{j 2}^{\prime} & \cdots & x_{2 i} x_{j n}^{\prime} \\
\vdots & \vdots & \vdots & \vdots \\
x_{n i} x_{j 1}^{\prime} & x_{n i} x_{j 2}^{\prime} & \cdots & x_{n i} x_{j n}^{\prime}
\end{array}\right) .
\end{aligned}
$$

Hence the element at the $k l$ th place of $\operatorname{Ad}(x)\left(e_{i j}\right)$ is

$$
x_{k i} x_{j l}^{\prime}=x_{k i} \bar{x}_{l j} \text {. }
$$

Lemma 5. Let $x \in \mathrm{SL}(n) . \operatorname{Ad}(x)$ is unitary if and only if $\operatorname{ad}(x) \in \operatorname{GL}(\mathfrak{s l}(n))$ is unitary.

Proof. Recall the split exact sequence $(* *)$ of GL $(n)$-modules. From it, we see that with respect to any unitary basis containing $\frac{1}{\sqrt{n}}\left(e_{11}+\cdots+e_{n n}\right), \operatorname{Ad}(x)$ is of the form

$$
\left(\begin{array}{cc}
1 & 0 \\
0 & \operatorname{ad}(x)
\end{array}\right)
$$

and it follows immediately that $\operatorname{Ad}(x)$ is unitary if and only if $\operatorname{ad}(x)$ is so.

Lemma 6. Let $x \in \mathrm{GL}(n)$. Then $\operatorname{Ad}(x)$ is unitary if and only if $x$ is so.

Proof. We have

$$
\begin{aligned}
\left(\operatorname{Ad}(x) \cdot \overline{\operatorname{Ad}(x)}^{T}\right)_{i j k l} & =\sum_{r, s}(\operatorname{Ad}(x))_{i j r s} \cdot\left(\overline{\operatorname{Ad}(x)}^{T}\right)_{r s k l} \\
& =\sum_{r, s}(\operatorname{Ad}(x))_{i j r s} \cdot \overline{\operatorname{Ad}(x)_{k l r s}} \\
& =\sum_{r, s} x_{i r} \bar{x}_{j s} \cdot \bar{x}_{k r} \bar{x}_{l s} \\
& =\sum_{r, s} x_{i r} \bar{x}_{j s} \cdot \bar{x}_{k r} x_{l s} \\
& =\left(x \cdot \bar{x}^{T}\right)_{i k} \cdot\left(x \cdot \bar{x}^{T}\right)_{l j} .
\end{aligned}
$$

Therefore, $\operatorname{Ad}(x)$ is unitary if and only if $(x x)=x \cdot \bar{x}^{T}$ is diagonal and $(x x)_{i i}$. $(x x)_{j j}=1$ for any $i, j$. Thus $(x x)_{i i}^{2}=1$, but $(x x)_{i i}=\sum_{s} x_{i s} \overline{x_{i s}} \geq 0$, and it follows that $(x x)$ is the identity matrix.

Combining Lemma 5 and Lemma 6, we obtain

Lemma 7. Let $x \in \mathrm{SU}(n)$. Then $\operatorname{ad}(x)$ is unitary. 
Example 1. In the rank 2 case $(x \in \mathrm{U}(2))$,

$$
\operatorname{Ad}(x)=\left(\begin{array}{llll}
x_{11} \bar{x}_{11} & x_{11} \bar{x}_{12} & x_{12} \bar{x}_{11} & x_{12} \bar{x}_{12} \\
x_{11} \bar{x}_{21} & x_{11} \bar{x}_{22} & x_{12} \bar{x}_{21} & x_{12} \bar{x}_{22} \\
x_{21} \bar{x}_{11} & x_{21} \bar{x}_{12} & x_{22} \bar{x}_{11} & x_{22} \bar{x}_{12} \\
x_{21} \bar{x}_{21} & x_{21} \bar{x}_{22} & x_{22} \bar{x}_{21} & x_{22} \bar{x}_{22}
\end{array}\right),
$$

and with respect to the unitary basis $\left\{e_{12}, e_{21}, \frac{1}{\sqrt{2}}\left(e_{11}-e_{22}\right)\right\}$,

$$
\operatorname{ad}(x)=\left(\begin{array}{ccc}
x_{11} \bar{x}_{22} & x_{12} \bar{x}_{21} & \sqrt{2} x_{11} \bar{x}_{21} \\
x_{21} \bar{x}_{12} & x_{22} \bar{x}_{11} & \sqrt{2} x_{21} \bar{x}_{11} \\
\sqrt{2} x_{11} \bar{x}_{12} & \sqrt{2} x_{12} \bar{x}_{11} & \left|x_{11}\right|^{2}-\left|x_{12}\right|^{2}
\end{array}\right) .
$$

Proposition 4. For any integer $g>2$, there exists an irreducible unitary representation $\rho$ of $\Gamma_{g}$ of rank 2 such that $\operatorname{ad}(\rho)$ is also irreducible and unitary.

Proof. Let $z$ be a complex number satisfying $z^{2} \neq \bar{z}^{2}$, and set $r=|z|$. Let

$$
A_{1}=\left(\begin{array}{cc}
0 & i \\
i & 0
\end{array}\right), B_{1}=\left(\begin{array}{cc}
i & 0 \\
0 & -i
\end{array}\right), B_{2}=\frac{1}{r \sqrt{2}}\left(\begin{array}{cc}
z & -\bar{z} \\
z & \bar{z}
\end{array}\right), A_{2}=B_{2}^{-1} .
$$

Also, let $A_{3}=A_{1}, B_{3}=B_{1}$, and $A_{i}=B_{i}=1$ for $i>3$. Then $\left[A_{1} B_{1}\right]=-1$, $\left[A_{2} B_{2}\right]=1,\left[A_{3} B_{3}\right]=-1$ and hence $\prod_{i=1}^{g}\left[A_{i} B_{i}\right]=1$.

With respect to the unitary basis $\left\{e_{1}^{\prime}=e_{12}, e_{2}^{\prime}=e_{21}, e_{3}^{\prime}=\frac{1}{\sqrt{2}}\left(e_{11}-e_{22}\right)\right\}$, we have

$$
\begin{gathered}
\operatorname{ad}\left(A_{1}\right)=\left(\begin{array}{ccc}
0 & 1 & 0 \\
1 & 0 & 0 \\
0 & 0 & -1
\end{array}\right), \\
\operatorname{ad}\left(B_{1}\right)=\left(\begin{array}{ccc}
-1 & 0 & 0 \\
0 & -1 & 0 \\
0 & 0 & 1
\end{array}\right), \\
\operatorname{ad}\left(B_{2}\right)=\frac{1}{2 r^{2}}\left(\begin{array}{ccc}
z^{2} & -\bar{z}^{2} & \sqrt{2} r^{2} \\
-z^{2} & \bar{z}^{2} & \sqrt{2} r^{2} \\
-\sqrt{2} z^{2} & -\sqrt{2} \bar{z}^{2} & 0
\end{array}\right) .
\end{gathered}
$$

We need to show that they do not stabilize any proper subspace. The eigenvectors of $\operatorname{ad}\left(A_{1}\right)$ are $\mathbb{C}\left(e_{1}^{\prime}+e_{2}^{\prime}\right), \mathbb{C}\left(e_{1}^{\prime}-e_{2}^{\prime}\right)+\mathbb{C} \cdot e_{3}^{\prime}$. Those of $\operatorname{ad}\left(B_{1}\right)$ are $\mathbb{C} \cdot e_{3}^{\prime}$ and $\mathbb{C} \cdot e_{1}^{\prime}+\mathbb{C} \cdot e_{2}^{\prime}$. Hence common eigenvectors for the two are $\mathbb{C} \cdot e_{3}^{\prime}, \mathbb{C} \cdot\left(e_{1}^{\prime}-e_{2}^{\prime}\right)$ and $\mathbb{C} \cdot\left(e_{1}^{\prime}+e_{2}^{\prime}\right)$. But none of them is an eigenvector for $\operatorname{ad}\left(B_{2}\right)$.

We need to show that there is no 2-dimensional subspace stabilized by the above three transformations. Assume that $W$ is such a subspace. Since $\operatorname{ad}\left(B_{2}\right)$ does not stabilize $\operatorname{Span}\left\{e_{1}^{\prime}, e_{2}^{\prime}\right\}, W$ contains an element $w=(a, b, c)=a e_{1}^{\prime}+b e_{2}^{\prime}+c e_{3}^{\prime}$ with $c \neq 0$. Consider the following cases.

1. $a=b=0$ : Then $W$ is spanned by $e_{3}^{\prime}$ and $\lambda e_{1}^{\prime}+\mu e_{2}^{\prime}$ for some $\lambda, \mu \in$ C. For $\operatorname{ad}\left(A_{1}\right)$ to stabilize $\operatorname{Span}\left\{e_{3}^{\prime}, \lambda e_{1}^{\prime}+\mu e_{2}^{\prime}\right\}$, either $\lambda=\mu$ or $\lambda=-\mu$. But Span $\left\{e_{3}^{\prime}, e_{1}^{\prime}+e_{2}^{\prime}\right\}$ and $\operatorname{Span}\left\{e_{3}^{\prime}, e_{1}^{\prime}-e_{2}^{\prime}\right\}$ are not stabilized by ad $\left(B_{2}\right)$, since

$$
\begin{aligned}
\operatorname{ad}\left(B_{2}\right)\left(e_{1}^{\prime}+e_{2}^{\prime}\right) & =\frac{1}{2 r^{2}}\left(z^{2}+\bar{z}^{2},-z^{2}+\bar{z}^{2},-\sqrt{2}\left(z^{2}-\bar{z}^{2}\right)\right) \\
& \notin \operatorname{Span}\left\{e_{1}^{\prime}+e_{2}^{\prime}, e_{3}^{\prime}\right\},
\end{aligned}
$$

and

$$
\operatorname{ad}\left(B_{2}\right)\left(e_{3}^{\prime}\right)=\frac{1}{2 r^{2}}\left(\sqrt{2} r^{2}, \sqrt{2} r^{2}, 0\right) \notin \operatorname{Span}\left\{e_{1}^{\prime}-e_{2}^{\prime}, e_{3}^{\prime}\right\}
$$


2. $a \neq 0$ : We can assume that $w$ is not an eigenvector of $\operatorname{ad}\left(A_{1}\right)$. (Otherwise $w$ is of the form $(1,-1, c)$ and $W$ is spanned by $(1,-1, c)$ and $\operatorname{ad}\left(B_{1}\right)(w)=(-1,1, c)$. That is, $W=\operatorname{Span}\left\{e_{1}^{\prime}-e_{2}^{\prime}, e_{3}^{\prime}\right\}$; but we have already seen in case 1 that this is not stabilized by $\operatorname{ad}\left(B_{2}\right)$.) Therefore, $W$ is spanned by $w$ and $w^{\prime}=\operatorname{ad}\left(A_{1}\right)(w)=$ $(b, a,-c)$. Since $\operatorname{ad}\left(B_{1}\right)(w)=(-a,-b, c)$ has to be in $W$, there are $x, y \in \mathbb{C}$ such that

$$
(-a,-b, c)=x w+y w^{\prime}=(x a+y b, x b+y a,(x-y) c) .
$$

From this we get $x-y=1$ and $(x+1)^{2}-y^{2}=0$, which forces $x=0, y=-1$, and $a=b$. But then $W$ is spanned by $w=(a, a, c)$ and $\operatorname{ad}\left(B_{1}\right)(w)=(-a,-a, c)$. So $W=\operatorname{Span}\left\{e_{1}^{\prime}+e_{2}^{\prime}, e_{3}^{\prime}\right\}$, which we have already seen is not stabilized by $\operatorname{ad}\left(B_{2}\right)$.

3. $a=0, b \neq 0$ : Then $W$ is spanned by $w=(0, b, c)$ and $w^{\prime}=\operatorname{ad}\left(A_{1}\right)(w)=$ $(b, 0,-c)$. Also,

$$
\operatorname{ad}\left(B_{1}\right)(w)=(0, b,-c)=x w+y w^{\prime}=(y b, x b,(x-y) c)
$$

implies $y=0$ and forces $(0,-b, c)=(0, b, c)$. Since $b \neq 0$, this is impossible.

Combining this with Proposition 3, we obtain

Proposition 5. There exist Ad-stable SL(2)-bundles over any compact Riemann surface of genus $>2$.

Example 2 (A unitary irreducible representation $\rho$ whose adjoint $\operatorname{Ad}(\rho)$ is not irreducible). Let

$$
A_{1}=\left(\begin{array}{cc}
i & 0 \\
0 & -i
\end{array}\right), A_{2}=\left(\begin{array}{cc}
0 & -1 \\
1 & 0
\end{array}\right),
$$

and let all $B_{i}$ and $A_{j}, j>2$, be 1 . Clearly $\prod_{i=1}^{g}\left[A_{i} B_{i}\right]=1$. They form an irreducible set, since they do not have any common eigenvector.

Now, let us take a look at the adjoint representation $\operatorname{Ad}(\rho): \Gamma_{g} \rightarrow G L(\mathfrak{g l}(2))$. We use the formula

$$
(\operatorname{Ad}(A))_{k l i j}=A_{k i} \overline{A_{l j}}
$$

with respect to the standard basis, and get

$$
\begin{aligned}
\operatorname{Ad}\left(A_{1}\right) & =\left(\begin{array}{cccc}
1 & 0 & 0 & 0 \\
0 & -1 & 0 & 0 \\
0 & 0 & -1 & 0 \\
0 & 0 & 0 & 1
\end{array}\right), \\
\operatorname{Ad}\left(A_{2}\right) & =\left(\begin{array}{cccc}
0 & 0 & 0 & 1 \\
0 & 0 & -1 & 0 \\
0 & -1 & 0 & 0 \\
1 & 0 & 0 & 0
\end{array}\right) .
\end{aligned}
$$

With respect to $\left\{e_{11}-e_{22}, e_{12}, e_{21}\right\}$, one has

$$
\operatorname{ad}\left(A_{1}\right)=\left(\begin{array}{ccc}
1 & 0 & 0 \\
0 & -1 & 0 \\
0 & 0 & -1
\end{array}\right) \text { and } \operatorname{ad}\left(A_{2}\right)=\left(\begin{array}{ccc}
-1 & 0 & 0 \\
0 & 0 & -1 \\
0 & -1 & 0
\end{array}\right),
$$

which is certainly reducible.

Remark 2. By Theorem 1 and Proposition 2, this example provides us with a stable $\mathrm{SL}(2)$-bundle that is not Ad-stable. 


\section{REFERENCES}

[1] B. Balaji and C. S. Seshadri, Semistable principal bundles, I, J. Algebra 258 (2002), 321-347. MR 2003m:14050

[2] R. Friedman and J. W. Morgan, Holomorphic principal bundles over elliptic curves, Preprint (1998), math.AG/9811130.

[3] P. Griffiths and J. Harris, Principles of Algebraic Geometry, John Wiley \& Sons, Inc. (1978). MR 80b:14001

[4] M. S. Narasimhan and C. S. Seshadri, Stable and unitary vector bundles on a compact Riemann surface, Ann. of Math. (2) 82 (1965), 540-567. MR 32:1725

[5] D. Hyeon, Principal bundles over a projective scheme, Trans. Amer. Math. Soc., 354 (2002), 1899-1908. MR 2003d:14013

[6] A. Ramanathan, Moduli for principal bundles over algebraic curves I, II, Proc. Indian Acad. Sci. (Math. Sci.), 106 (1996), 301-328 and 421-449. MR 98b:14009a; MR 98b:14009b

[7] A. Ramanathan, Stable principal bundles on a compact Riemann surface, Math. Ann. 213 (1975), 129-152. MR 51:5979

Department of Mathematics, University of Illinois, 1409 W. Green Street, Urbana, ILLINOIS 61801

Current address: Department of Mathematics, Rice University, 6100 Main St., Houston, Texas 77005

E-mail address: hyeon@math.rice.edu

Department of Mathematics, University of Illinois, 1409 W. Green Street, Urbana, ILLINOIS 61801

E-mail address: dcmurphy@math.uiuc.edu 